\title{
Treatment Strategy and Safety of Eribulin in Advanced Breast Cancer
}

\author{
Xinli Wang, Shusong Liu and Yan Xue \\ Department of Oncology, Xi'an International Medical Center Hospital, Xi'an, Shaanxi, China
}

\begin{abstract}
This study sought to investigate the efficacy and safety of eribulin in advanced breast cancer patients. Eighteen patients with advanced breast cancer were retrospectively included. The previous use of taxanes, and the use of eribulin in subsequent treatment was reviewed. Progression- free survival $\square$ PFS $\square$ and the efficacy of eribulin, influencing factors, and adverse reactions were analysed. For patients who were previously treated with taxanes were more resistant, subsequent eribulin treatment chemotherapy PFS was generally for two months. However, for patients who chose eribulin combined with anlotinib for the latter-line regimen, PFS reached for five months, and these patients were in continuous remission. Among the 18 patients, 17 patients (94.4\%) had different degrees of bone marrow suppression. Patients who have not been treated with taxanes or who have been treated with taxanes for a long response time, experience better effects when eribulin was used as subsequent treatment. Eribulin combined with anlotinib is the preferred combination therapy.
\end{abstract}

Key Words: Breast cancer, Eribulin, Efficacy, Safety.

How to cite this article: Wang X, Liu S, Xue Y. Treatment Strategy and Safety of Eribulin in Advanced Breast Cancer. J Coll Physicians Surg Pak 2022; 32(01):122-124.

Female breast cancer has surpassed lung cancer and become the most common cancer in women worldwide. ${ }^{1}$ Chemotherapy still plays an important role in advanced breast cancer. After 20 years of the use of anthracyclines and taxanes as chemotherapeutic agents, few chemotherapeutic drugs offer OS benefits as single agents; and there is no clear standard for the selection of chemotherapy regimens after the application of anthracyclines and taxanes. Eribulin binds to the microtubule terminal with a highaffinity, causing irreversiblemitotic blockage and cell apoptosis and death. Eribulin is a microtubule kinetics inhibitor that differs from anthracene, taxanes and other chemotherapeutic drugs. At present, clinical practice has demonstrated that eribulin can be used as an important treatment option for patients with anthracene and post-taxane advanced breast cancer. Eribulin is also the only chemotherapy drug that has been evaluated by the National Comprehensive Cancer Network (NCCN) that demonstrates the same efficacy as anthracycline and taxol, breaking the pattern of chemotherapy drugs foradvanced breast cancer.

Correspondence to: Yan Xue, Department of Oncology, Xi'an International Medical Center Hospital, Xi'an, Shaanxi, China

E-mail: wshirly99@126.com

Received: June 17, 2021; Revised: October 13, 2021;

Accepted: November 09, 2021

DOI: https://doi.org/10.29271/jcpsp.2022.01.122
Although eribulin has been widely used in patients with metastatic breast cancer, the potential benefit population of eribulin and the best treatment options remain uncertain and need to be explored in the real world. Real-world studies can evaluate the effectiveness of specific drugs outside clinical trials. Although eribulin is currently widely used in patients with metastatic breast cancer, further evaluation of its efficacy and safety in real-world patients is necessary.

The purpose of this study was to evaluate the efficacy and safety of eribulin as a chemotherapeutic agent in advanced breast cancer, to lay a foundation for better understanding of the clinical application of eribulin, to determine the patient groups who will benefit most and the optimal treatment plan for eribulin, and to guide clinical treatment.

A retrospective analysis was conducted on the data of 18 patients with advanced breast cancer admitted to Xi'an International Medical Center Hospital from January 2020 to January 2021. The analysed data included age, histological type, molecular type, number of previous treatment lines, and previous use of taxanes and eribulin therapy. Inclusion criteria were: advanced breast cancer diagnosed histopathologically in patients who had received eribulin chemotherapy in the late stage. The exclusion criteria were breast cancer complications with other malignant tumours and incomplete data. Observation indices were progression-free survival (PFS), and drug-related adverse reactions (ADRs).

SPSS version 20.0 statistical software was used for data processing. The PFS between the two groups was compared, and the survival rate was estimated using the Kaplan-Meier method. 
Table I: Clinicopathological features and prognosis of the patients.

\begin{tabular}{|c|c|c|c|c|c|c|c|}
\hline Patient & $\begin{array}{c}\text { Age } \\
\text { (year) }\end{array}$ & Histological type & Molecular typing & $\begin{array}{c}\text { The number of previous } \\
\text { chemotherapy lines in } \\
\text { the late stage }\end{array}$ & $\begin{array}{l}\text { The previous use of taxanes } \\
{ }^{*} \text { Cycles in late stage }\end{array}$ & Eribulin *cycle & $\begin{array}{c}\text { PFS } \\
\text { (months) }\end{array}$ \\
\hline 1 & 41 & Infiltrating ductal carcinoma & Luminal A & 4 lines & $\begin{array}{c}\text { Docetaxel } * 5 \\
\text { Paclitaxel liposome } * 4\end{array}$ & $\begin{array}{c}\text { Eribulin } * 8 \\
(\text { Eribulin }+ \text { anlotinib }) * 2\end{array}$ & $6 \#$ \\
\hline 2 & 47 & Infiltrating ductal carcinoma & Luminal BᄆHer-2(-) & 1 line & No & Eribulin $* 4$ & 3 \\
\hline 3 & 40 & Infiltrating ductal carcinoma & Luminal A & 3 lines & $\begin{array}{c}\text { Docetaxel } * 5 \\
\text { Paclitaxel (albumin bound) } * 4 \\
\end{array}$ & Eribulin $* 2$ & 2 \\
\hline 4 & 42 & Infiltrating ductal carcinoma & Her-2(+) & 4 lines & Paclitaxel (albumin bound) $* 3$ & $($ Eribulin + nedaplatin $) * 2$ & 2 \\
\hline 5 & 28 & Infiltrating ductal carcinoma & Luminal B $\square$ Her-2(-) & 1 line & Paclitaxel (albumin bound) $* 5$ & (Eribulin + capecitabine) $* 2$ & 2 \\
\hline 6 & 54 & Infiltrating ductal carcinoma & Triple negative & 1 line & Paclitaxel (albumin bound) $* 6$ & (Eribulin + capecitabine $) * 2$ & 2 \\
\hline 7 & 70 & Infiltrating ductal carcinoma & Luminal B,Her-2(+) & 3 lines & No & Eribulin $* 8$ & 7\# \\
\hline 8 & 45 & Invasive lobular carcinoma & Luminal B $\square$ Her-2(-) & 2 lines & $\begin{array}{c}\text { Paclitaxel liposome } * 11 \\
\text { Docetaxel } * 9\end{array}$ & Eribulin $* 2$ & 2 \\
\hline 9 & 50 & Infiltrating ductal carcinoma & Her-2(+) & 4 lines & Paclitaxel (albumin bound) *7 & Eribulin $* 4$ & 3 \\
\hline 10 & 60 & Infiltrating ductal carcinoma & Luminal B $\square$ Her-2(-) & 3 lines & Paclitaxel (albumin bound) *6 & $($ Eribulin + anlotinib $) * 6$ & 5\# \\
\hline 11 & 43 & Infiltrating ductal carcinoma & Luminal B $\square$ Her-2(-) & 5 lines & Paclitaxel (albumin bound) $* 6$ & (Eribulin + anlotinib)*6 & $5 \#$ \\
\hline 12 & 55 & Infiltrating ductal carcinoma & Her-2(+) & 1 line & No & Eribulin $* 4$ & $4 \#$ \\
\hline 13 & 56 & Infiltrating ductal carcinoma & Triple negative & 2 lines & No & $($ Eribulin + anlotinib)*8 & $8 \#$ \\
\hline 14 & 63 & Infiltrating ductal carcinoma & Her-2(+) & 1 line & Docetaxel $* 4$ & $($ Eribulin + anlotinib)*6 & 5\# \\
\hline 15 & 58 & Infiltrating ductal carcinoma & Luminal B $\square$ Her-2(-) & 4 lines & Paclitaxel (albumin bound) $* 6$ & Eribulin $* 4$ & 3 \\
\hline 16 & 53 & Infiltrating ductal carcinoma & Triple negative & 3 lines & Paclitaxel (albumin bound) $* 4$ & $($ Eribulin + anlotinib $) * 5$ & 4 \\
\hline 17 & 49 & Infiltrating ductal carcinoma & Triple negative & 4 lines & Paclitaxel (albumin bound)*6 & Eribulin $* 4$ & 4 \\
\hline 18 & 51 & Infiltrating ductal carcinoma & Luminal BᄆHer-2(-) & 2 lines & No & $($ Eribulin + anlotinib $) * 8$ & 7\# \\
\hline
\end{tabular}

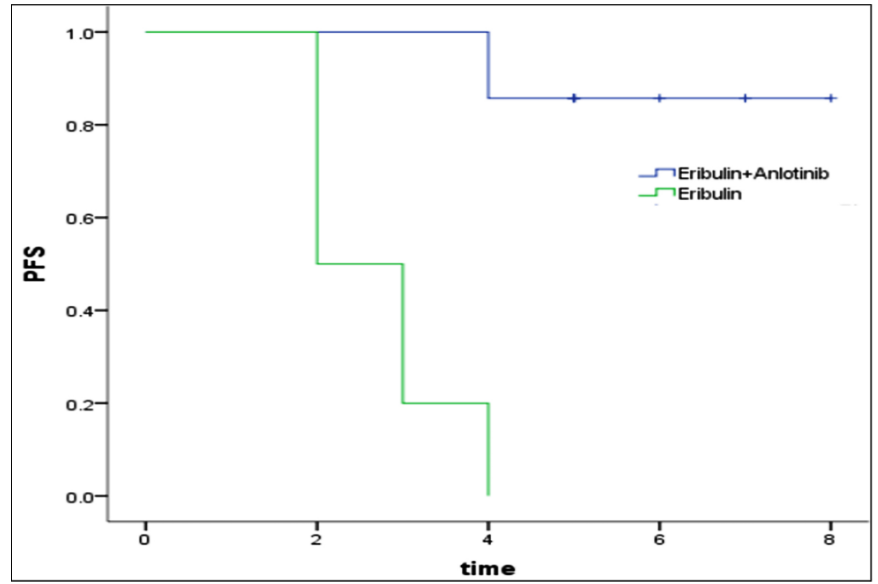

Figure 1: PSF of eribulin combined with anlotinib and eribulin.

A total of 18 patients with a median age of 50.5 years were included in this study. Among them, the remission time of the first patient was four years after treatment with five cycles of docetaxel after relapse and treatment with four cycles of paclitaxel liposomes after disease progression.

After treatment, the patient was in a stable condition, but in pursuit of better efficacy, the patient changed the regimen to eight cycles of eribulin and two cycles of eribulin; combined with anlotinib, the patient obtained continuous remission. Patients 3-6, 8, and 15-17 had previously used multicycle taxanes. These patients experienced disease progression during treatment as these patients developed resistance to taxanes. After treatment, patients were treated with eribulin chemotherapy, and progression typically occurred two cycles later with PFS values of 2-4 months. The time was short. The seventh patient, who had not previously received taxol chemotherapy, followed by seven months of PFS with eribulin chemotherapy, and the remission time was significantly prolonged. The 12th patient, who had not previously received taxane chemotherapy, was in continuous remission after four cycles of eribulin. The 10th and 11th patients previously received six cycles of albumin paclitaxel chemotherapy, and the 14th patient previously received four cycles of docetaxel chemotherapy. After progression, these three patients were switched to eribulin, combined with anlotinib treatment; and are still in continuous remission with five months of PFS to date. The 13th and 18th patients, who had not used taxanes in the advanced stage, were converted to eribulin combined with anlotinib after previous chemotherapy progression; and their PFS values were eight months and seven months, respectively. These patients remain in continuous remission (Table I).

Seventeen patients (94.4\%) developed varying degrees of myelosuppression after eribulin treatment, most of which were grade II-IV myelosuppression; six patients developed fatigue; seven patients developed mild liver function abnormalities; two patients developed renal function abnormalities; and two patients developed hair loss. One patient developed rashes and oral ulcer.

Follow-up for survival analysis ended June 30,2021 , with a median of five months (3.8 to 6.3 months). The median $(\mathrm{m})$ PFS of the two groups were different. The mPFS of the eribulin group was three months, and that of the eribulin combined with anlotinib group is not reached (NA) yet.

The mPFS in the eribulin combined with anlotinib group was longer than that in the eribulin monotherapy group (NA versus three months, $\mathrm{HR}=0.10,95 \% \mathrm{Cl}: 0.012$ to $0.821, \mathrm{p}$ $=0.006$, Figure 1 ).

The treatment choices for patients with advanced breast cancer following the administration chemotherapy are more limited. Due to the progress of patients on anthracene/taxane treatment, more effective and safer chemotherapy agents are urgently needed clinically. These agents are needed for post-resistance treatment to offer longer survival benefits for advanced breast cancer patients.

In the last 10 years, compared with the rapid progress of targeted therapy and endocrine therapy, the research and development of new drugs in the field of chemotherapy 
reached a bottleneck. Compared with traditional chemotherapy drugs, eribulin has significant clinical benefit of PFS. In addition to that, eribulin is the only new chemotherapy drug with an overall survival (OS) benefit in single-agent head-to-head phase III chemotherapy studies in the past 20 years, following the use of anthracyclines and taxol. ${ }^{2}$

Anlotinib is an small-molecule multitarget tyrosine kinase inhibitor with significant inhibitory activity against vascular endothelial growth factor receptor, platelet-derived growth factor receptor and FGF receptor. Antiangiogenic drugs can normalise tumor blood vessels, reduce vascular permeability, increase microcirculation oxygen content, and then increase chemotherapy drug perfusion, improve the efficacy of chemotherapy. A number of clinical trials have proved that anti-angiogenic drugs, combined with chemotherapy, can improve the efficacy of chemotherapy and bring benefits to patients. ${ }^{3-5}$ The mPFS in the eribulin, combined with anlotinib group, was longer than that in the eribulin monotherapy group (NA versus three months, $\mathrm{P}=0.006$ ). Our results also suggest that antiangiogenic agents can enhance the efficacy of chemotherapy.

The results show that the application of eribulin in advanced breast cancer patients with taxol resistance results in considerable progression after two cycles. The research results show that patients treated with taxol-based drugs without resistance may receive longer treatment benefits when eribulin is used as a posterior-line agent. The administration of eribulin with antiangiogenetic agents is an optional treatment regimen. The most common adverse reaction of eribulin was bone marrow suppression, and the remaining adverse safety events were grades 1 to 2 . In general, the safety of eribulin was controllable.

The results of this study can guide the clinical treatment of advanced breast cancer with eribulin to a certain extent, but this study is an exploratory analysis with a small sample. Thus, further studies with large sample sizes are needed.

\section{CONCLUSION}

Patients who have been treated with taxanes and were more resistant and subsequently treated with eribulin, typically progress after two cycles. In advanced breast cancer, eribulin may be more effective in the frontline, and the combination with antiangiogenic drugs can improve the efficacy. Myelosuppression was the most common adverse event of eribulin.

\section{ETHICAL APPROVAL:}

This clinical study is a retrospective study, only collecting patients' clinical data without interfering with patients' treatment plan, which will not bring physiological risks to patients. The authors didn't reveal personal privacy. Thus, this study was exempted by the Ethics Committee of Xi'an International Medical Center Hospital.

\section{CONFLICT OF INTEREST:}

The authors declared no conflict of interest.

\section{AUTHORS' CONTRIBUTION:}

$X W$ : Designed the study, collected the data, and wrote the paper.

SL: Contributed to data analysis.

YX: Edited the paper and revised the paper.

All authors reviewed the paper and approved the final manuscript.

\section{REFERENCES}

1. Siegel RL, Miller KD, Fuchs HE, Jemal A. Cancer Statistics, 2021. CA Cancer J Clin 2021; 71(1):7-33. doi: 10.3322/caac. 21654.

2. Cortes J, O'Shaughnessy J, Loesch D. Eribulin monotherapy versus treatment of physician's choice in patients with metastatic breast cancer (EMBRACE): A phase 3 open-label randomised study. Lancet 2011; 377(9769):914-23. Doi: 10.1016/S0140-6736(11)60070-6.

3. Wu YS, Shui L, Shen D. Bevacizumab combined with chemotherapy for ovarian cancer an updated systematic review and meta-analysis of randomised controlled trials. Oncotarget 2017; 8(6):10703-13. doi: 10.18632/oncotarget. 12926.

4. Li X, Gu G, Soliman F. The evaluation of durative transfusion of endostar combined with chemotherapy in patients with advanced non-small cell lung cancer. Chemotherapy 2018; 63(4): 214-9. doi: 10.1159/000493098.

5. Cheng H, Sun A, Guo Q. Emicacy and safety of apatinib combined with chemotherapy for the treatment of advanced gastric cancer in the Chinese population: A systematic review and meta-analysis. Drug Des Devel Ther 2018; 12:2173-83. doi: 10.2147/DDDT.S170678. 